\title{
POF over WDM—Beyond the Dispersion Limit
}

\author{
U. H. P. Fischer ${ }^{1}$, S. Höll ${ }^{2}$, M. Haupt ${ }^{1}$ and M. Joncic ${ }^{1}$ \\ 1. Harz University of Applied Sciences, Wernigerode 38855, Germany \\ 2. Sicoya GmbH, Berlin 12489, Germany
}

\begin{abstract}
Data communication over POFs (polymer optical fibers) is limited to only one channel for data transmission. Therefore the bandwidth is strongly restricted. By using more than one channel, it is possible to break through the limit. This technique is called WDM (wavelength division multiplexing). It uses different wavelengths in the visible spectrum to transmit data parallel over one fiber. Two components are essential for this technology: A MUX (multiplexer) and a DEMUX (demultiplexer). The multiplexer collects the light of the different sources to one fiber and the demultiplexer separates the light at the end of the fiber into the different fiber output ports. In this paper, we propose spectral grids in the visible spectrum, which are compatible with existing standards like ITU recommendations for WDM networks. Additionally, results of a demonstrator of an integrated polymeric demultiplexer produced with injection molding are presented. The paper discusses the results of the different development steps, the measurements done with the first demonstrator and the challenges related to the injection molding process.
\end{abstract}

Key words: POF, WDM, (de-)multiplexer, spectral grid, injection molding.

\section{Introduction}

POFs (polymer optical fibers) are used in various fields of applications. The core material consists of PMMA (polymethylmethacrylate), while the cover is made of fluorinated PMMA. The whole fiber has a diameter of $1 \mathrm{~mm}$. POFs are used for optical data transmission based on the same principle as glass fiber. As a communication medium they offer a couple of advantages related to other data communication systems such as copper cables, glass fibers and wireless systems, and have great potential to replace them in different applications.

Namely, in comparison GOF (with glass fibers), POFs have the advantage of easy and economical processing and are more flexible for optical connections [1]. However, one advantage of using glass fibers is their low attenuation, which is below $0.2 \mathrm{~dB} / \mathrm{km}$ in the infrared range. The larger core diameter of POFs leads to higher mode dispersion and thus to higher attenuation across the electromagnetic spectrum. This increased attenuation leaves only one remaining transmission

Corresponding author: U. H. P. Fischer, Dr., Prof., research fields: photonic communications, photonic packaging. window, namely the visible spectrum of light (400-700 $\mathrm{nm})$. Hence, POFs are best suited for the use in short distance data communication. Here, POFs can outperform the current standard of copper cable as communication medium. On the one hand, they feature lower weight and space. On the other hand, POFs are not susceptible to electromagnetic interference [2, 3]. For these reasons, POFs are already used in various application domains, for example in the automotive sector and for in-house communication [4-7]. At present, the great potential of the POF is not available as the alternative techniques offer transmission rates up to 10 Gb over copper and up to $40 \mathrm{~Gb}$ over glass fibers in the network area. The WDM technique offers an approach to achieve these high data rates also in the POF range.

For WDM two essential components are needed: A MUX (multiplexer) and a DEMUX (demultiplexer). To create a functional demultiplexer for POF, several preconditions must be fulfilled. Firstly, a mirror must focus the divergent light beam coming from the POF. The shape of this mirror cannot be spherical because of the appearing spherical aberration. Instead, a toric shape of the mirror prevents spherical aberration [8-10].

The second function is the separation of the different 
transmitted wavelengths, which can be achieved by a diffraction grating.

\section{Optical Simulations}

The principle of a (de-)multiplexer is illustrated in Fig. 1. The light is split into different orders of diffraction. The first order is the important one to regain all information. Ray tracing simulations were performed to analyze the way of light and to optimize the shape of this optical element [8-10].

Without a grating, a perfect point to point mapping (without any aberrations) is possible with an ellipsoid mirror because of the two foci, but there is no separation of the different channels. With a grating stamped on the mirror, the separation of the multicolored light in its monochromatic parts is possible. But this grating distorts the optical path of light dramatically.

The first change is that the gap of the different colors in the image layer (here the POF- or detector-array) increases with the line density of the grating. This can be noticed for an ellipsoid mirror and for a spherical mirror as well. The advantage of the spherical mirror is that it can be manufactured more easily for the injection molding process. This optimization process was shown in different papers before Refs. [9, 10].

The result so far is that the key-element can separate up to eight colors with enough space in between to regain the information with a POF- or detector-array.

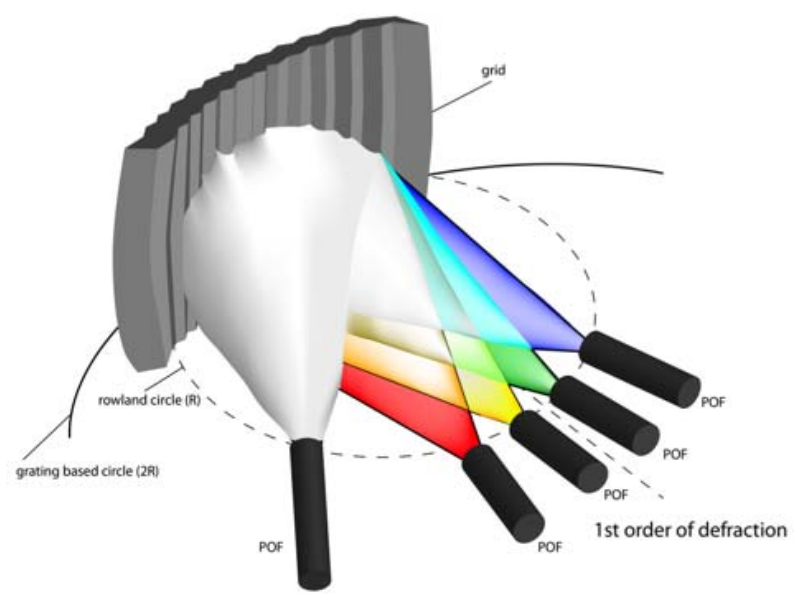

Fig. 1 Rowland set-up of demultiplexer.
The shapes of the foci feature low coupling losses and the shape of the mirror is producible in injection molding.

\section{Manufacturing of the Demonstrator}

There, the outgoing POFs can be placed. To separate the channels at the output ports, one interesting option for high multimode transmission systems is to use an optical grating. Here, the optical grating is placed on an aspheric mirror, which focuses the monochromatic parts of light into the outgoing fibers. In order to keep the advantage of cost-effective POFs it is necessary to mass-produce the MUX and DEMUX component at reasonable prices. For polymers, injection molding is the only technology, which offers high potential to achieve his goal. Before starting the production of the mold insert, a demonstrator of the DEMUX is fabricated by directly machining it in the PMMA material by means of diamond turning technique (see Fig. 2). Thus, the same diamond-turning technology is used for the manufacture of the mold insert. This step is done due to validating the simulation results with the produced component.

By using the injection molding process, the manufacturing of the mold insert is the most important factor. Due to the three-dimensional toric structure of the grating planar manufacturing methods like lithography, especially LIGA (German acronym for

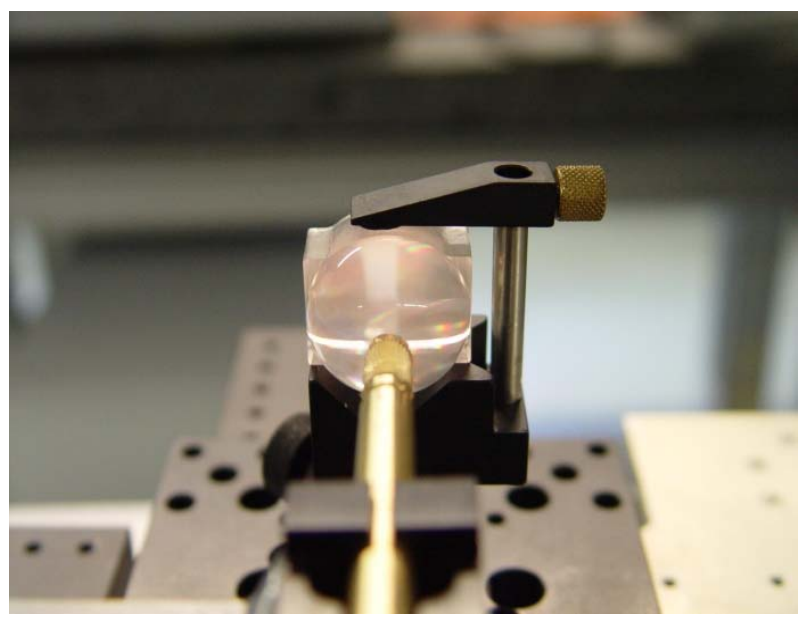

Fig. 2 Integrated demultiplexer prototype. 
Lithographie, Galvanoformung, AbformungLithography, Electroplating, and Molding) cannot be used. LIGA is used to manufacture planar spectrometers based on the glass fiber technology [11-14]. But in this case, the three-dimensional grating needs another machining method. Especially the microstructure of the grating and the exact curve shape of the toric surface require high precision. The microstructure has the shape of a saw tooth with a pitch between the teeth of $2.5 \mu \mathrm{m}$. Fig. 3 shows an enlarged 3D-Model of the grating. After investigating several machining methods only the diamond turning meets the high demands of the micro structured grating.

\section{Optical Measurements}

In order to measure the position of the focal points of the different separated wavelength a special measurement setup was build. It uses a parallel-kinematics precision alignment system to align a fiber at the surface of the hemisphere (see Fig. 4). A fixed input-fiber is used to couple light (first from a white light source and second from RGB laser diodes) into the DEMUX as shown in Fig. 5. In this figure it can be seen that the separated wavelength is focused at a ring on the hemisphere (see also Fig. 6). This ring is scanned by the fiber on the alignment system. A Thorlabs S140C integrating sphere and a PM100D optical power meter were used to measure the transmitted power at the end of the output fiber. The

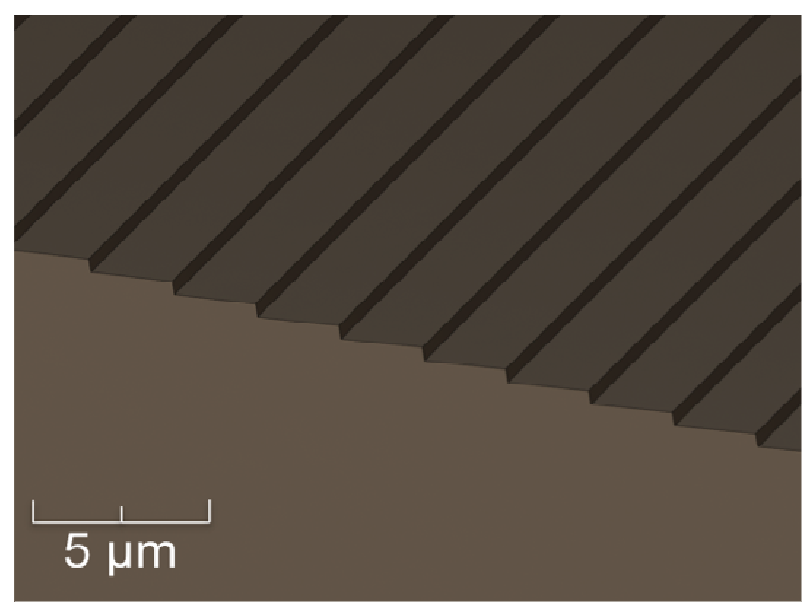

Fig. 3 Grating of the demultiplexer.

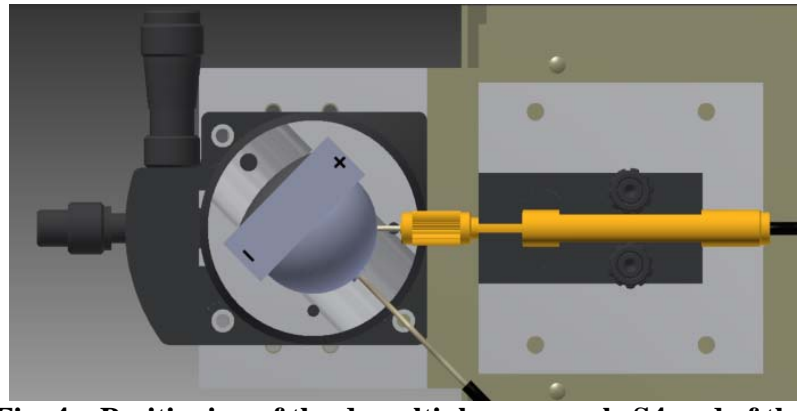

Fig. 4 Positioning of the demultiplexer sample S4 and of the output fiber.

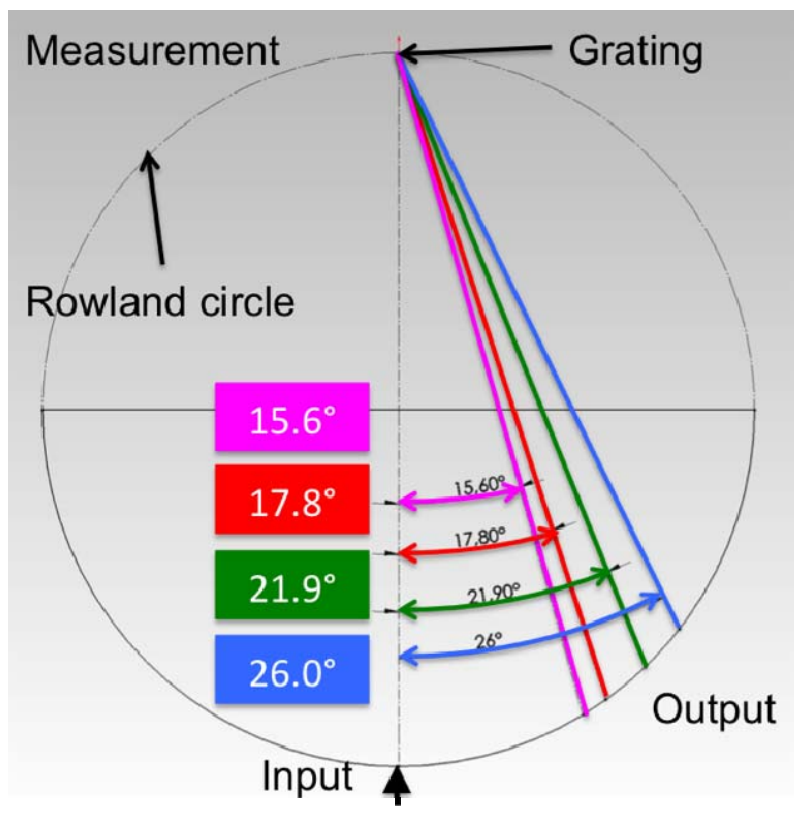

Fig. 5 Measurement results of the focal points for different wavelengths (405 nm violet ray; $450 \mathrm{~nm}$ blue ray; $520 \mathrm{~nm}$ green ray; $650 \mathrm{~nm}$ red ray).

positions of the wavelengths measured by the setup are depicted in Fig. 5. In comparison to the simulation a shift of the positions can be recognized. Nevertheless, the separation of the wavelengths was measured and confirmed the functionality of the demultiplexer.

\section{Spectral Grids in the Visible Spectrum for POF WDM Applications}

Besides developing low-IL cost-effective POF WDM components and fast POF WDM transmission systems, it is also important to allocate a unique set of WDM transmission channels in the visible spectrum to support WDM applications over SI-POF. To evaluate the applicability of a spectral grid to support visible 
spectrum WDM applications over SI-POF, the appropriate criteria were first established. Those criteria refer to:

- Channel distribution with respect to the spectral attenuation of SI-POF;

- Performances of different demultiplexing techniques;

- Availability of laser diodes in the visible spectrum.

\subsection{Extension of ITU-T G.694.2 CWDM Grid into the Visible Spectrum}

If ITU-T G.694.2 CWDM wavelength grid would be extended into the visible spectrum, 15 equidistant channels between $400 \mathrm{~nm}$ and $700 \mathrm{~nm}$ would be obtained, as shown in Fig. 7. The parameters of the grid include the nominal central wavelengths. The channel

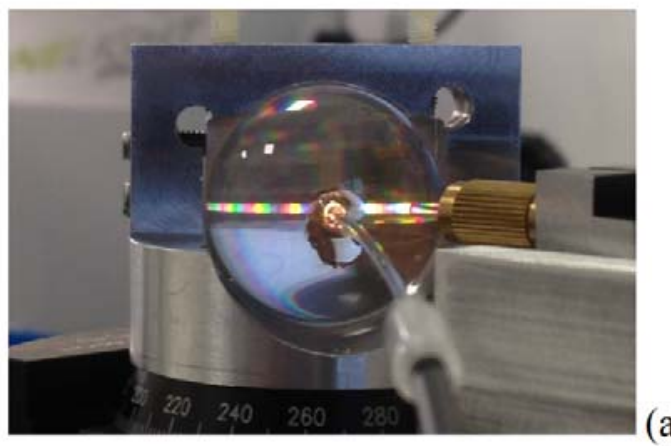

(a) spacing of $20 \mathrm{~nm}$ makes good utilization of the available spectral range. In the red window the extension has a channel at $651 \mathrm{~nm}$, which is very close to the attenuation minimum at $650 \mathrm{~nm}$. The channel distribution also corresponds well to three other attenuation windows. The channels experiencing the highest attenuation are those at $611 \mathrm{~nm}, 631 \mathrm{~nm}, 671 \mathrm{~nm}$ and $691 \mathrm{~nm}$. Those channels could be used for distances up to $20 \mathrm{~m}$ since they would experience approximately the same attenuation as $651 \mathrm{~nm}$ channel over $50 \mathrm{~m}$, but lower intermodal dispersion. Good channel allocation, sufficient channel spacing, high channel count and good availability of the transmitters make the extension of CWDM grid very suitable to support WDM applications over SI-POF.

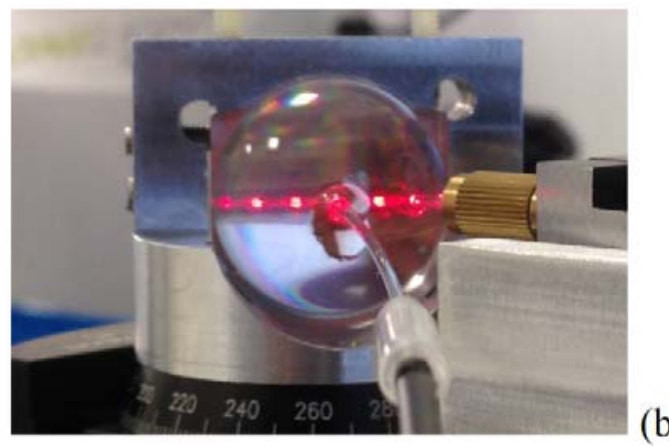

Fig. 6 Measuring transmission properties of the demultiplexer when launching with (a) white light source and (b) $638 \mathrm{~nm}$ laser diode.

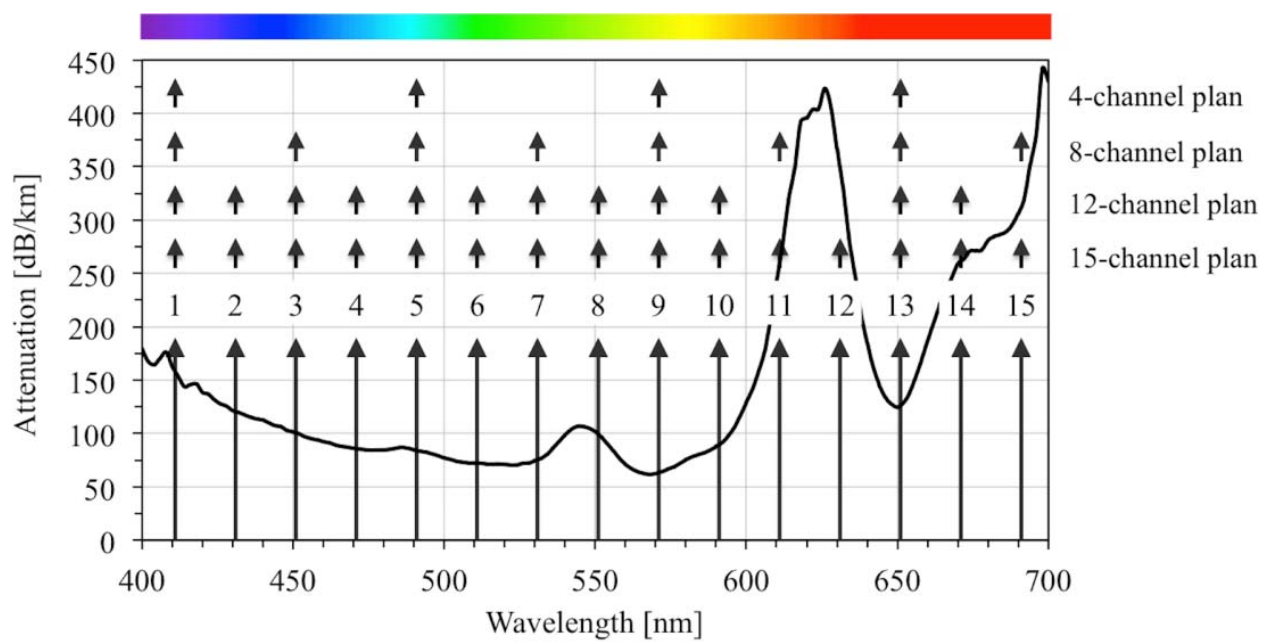

Fig. 7 Extension of CWDM wavelength grid into the visible spectrum and channel plans for 4-, 8-, 12- and 15-channel applications. 


\section{Conclusions}

In summary, it is shown that WDM can also be applied in the visible range for POF communication systems. The design and development of the key-element for WDM, a (de-)multiplexer, was presented. This optical component could open mass market if produced in injection molding. But this technology is challenging, especially the microstructure of the grating on a three-dimensional surface. It is shown that the current manufacturing process is able to produce the structure size and the exact radius needed for the DEMUX. The optical measurements to prove the principle were done. These confirm the separation of the wavelength in the visible spectrum.

A wavelength grid based on CWDM in IR was presented. In the near future, WDM applications over SI-POF with $40 \mathrm{Gbit} / \mathrm{s}$ transmissions are a realistic aim for developments in POF systems. It seems to be possible to transmit $40 \mathrm{Gbit} / \mathrm{s}$ via 15 channels and a channel rate of $2.7 \mathrm{Gbit} / \mathrm{s}$ as an overall data rate with WDM. This opens the range of POF applications to existing cloud centers and future in-house networks with extensions to $100 \mathrm{~m}$-link length.

\section{Acknowledgements}

We gratefully acknowledge the funding by the German Ministry of Education and Research (BMBF) under grant number 16V0009 (HS Harz)/16V0010 (TU BS) and program "Zwanzig 20-Partnerschaft für Innovation” contract no. 03ZZ0519I. All injection moulded parts are done with the support of the Institute of Micro and Sensor Systems at the Otto-von-Guericke University Magdeburg and Prof. Bertram Schmidt.

\section{References}

[1] Daum, W., Krauser, J., Zamzow, P. E., and Ziemann, O. 2008. POF Handbook: Optical Short Range Transmission
Systems. Springer-Verlag.

[2] Nalwa, H. S., ed. 2004. Polymer Optical Fibres. California: American Scientific Publishers.

[3] Marcou, J. 1997. Club des Fibres Optiques Plastiques (CFOP) France, Plastic Optical Fibres-Practical Applications. John Wiley \& Sons.

[4] Brandrup, J., Immergut, E. H., and Grulke, E. A. 1999. Polymer Handbook. 4th Edition, Wiley-Interscience.

[5] Chen, R. T., and Lipscomb, G. F., eds. 2000. "WDM and Photonic Switching Devices for Network Applications.” In Proceedings of SPIE, vol. 3949.

[6] Colachino, J. 2001. "Mux/DeMux Optical Specifications and Measurements." Lightchip Inc. White Paper, Lightreading.

[7] Gnauck, H., Chraplyvy, A. R., Tkach, R. W., Zyskind, J. L., Sulhoff, J. W., Lucero, A. J., et al. 1996. "One Terabit/s Transmission Experiment.” Proceedings OFC'96.

[8] Fischer-Hirchert, U. H. P. 2015. "Photonic Packaging Sourcebook: Fiber-Chip Coupling for Optical Components, Basic Calculations, Modules.”

[9] Fischer, U. H. P., and Haupt, M. 2007. "WDM over POF: The Inexpensive Way to Break-through the limitation of Bandwidth of Standard POF Communication." SPIE Symposium on Integrated Optoelectronic Devices, Photonics West San Jose.

[10] Fischer, U. H. P., and Haupt, M. 2010. "Integrated WDM System for POF Communication with Low Cost Injection Moulded Key Components.” Access Networks and In-house Communications.

[11] Stricker, M., Pillwein, G., and Giessauf, J. 2009. "Focus on Precision-Injection Molding Optical Components.” In Kunststoffe International 4: 15-9.

[12] Ferguson, J. P., and Schoenfelder, S. 1999. "Micromoulded Spectrometers Produced by the Liga Process.” Searching for Information: Artificial Intelligence and Information Retrieval Approaches, IEE Two-day Seminar (Ref. No. 1999/199), 11/1-11/4.

[13] Davies, M. A., Evans, C. J., Vohra, R. R., Bergner, B. C., and Patterson, S. R. 2003. "Application of Precision Diamond Machining to the Manufacture of Microphotonics Components.” Proc. SPIE 5183, Lithographic and Micromachining Techniques for Optical Component Fabrication II.

[14] Dornfeld, D., Min, S., and Takeuchi, Y. 2006. "Recent Advances in Mechanical Micromachining.” CIRP Annals-Manufacturing Technology 55 (2): 7. 